\title{
Observation of collective decay dynamics of a single Rydberg superatom
}

\author{
Nina Stiesdal $\odot,{ }^{1}$ Hannes Busche $\odot,{ }^{1}$ Jan Kumlin $\odot,{ }^{2}$ Kevin Kleinbeck, ${ }^{2}$ Hans Peter Büchler $\odot,{ }^{2}$ and Sebastian Hofferberth $\odot$ 1,* \\ ${ }^{1}$ Department of Physics, Chemistry and Pharmacy, Physics@SDU, University of Southern Denmark, 5320 Odense, Denmark \\ ${ }^{2}$ Institute for Theoretical Physics III and Center for Integrated Quantum Science and Technology, University of Stuttgart, \\ 70550 Stuttgart, Germany
}

(Received 11 May 2020; accepted 11 November 2020; published 8 December 2020)

\begin{abstract}
We experimentally investigate the collective decay of a single Rydberg superatom, formed by an ensemble of thousands of individual atoms supporting only a single excitation due to the Rydberg blockade. Instead of observing a constant decay rate determined by the collective coupling strength to the driving field, we show that the enhanced emission of the single stored photon into the forward direction of the coupled optical mode depends on the dynamics of the superatom before the decay. We find that the observed decay rates are reproduced by an expanded model of the superatom which includes coherent coupling between the collective bright state and subradiant states.
\end{abstract}

DOI: 10.1103/PhysRevResearch.2.043339

\section{INTRODUCTION}

The collective interaction between an ensemble of emitters and photons is a fundamental topic of quantum optics, which has been extensively studied for over 60 years [1]. Collective enhancement of the emission, known as superradiance, has been observed in a variety of physical systems ranging from atoms [2] and ions [3] over molecules [4], artificial atoms coupled to microwave waveguides [5], and solid-state systems [6,7], to ensembles of nuclei [8]. Suppression of emission is more elusive because excitation fields typically do not couple to subradiant states, and was only recently observed for ensembles of more than two emitters [9,10]. Here, we investigate the collective emission of a single photon from a Rydberg superatom [11] and show that the experimentally observed decay rate depends on the initial-state preparation by a few-photon driving field [12]. We attribute this effect to a coherent population redistribution between collective superand subradiant states due to coherent excitation exchange between the individual emitters inside the superatom $[13,14]$.

A collectively excited ensemble features modifications to the rate and spatial distribution of its spontaneous emission [15-18], and coherent exchange of photons between individual emitters in an ensemble results in a collective Lamb shift [8,19-24]. These phenomena can be understood in a semiclassical approach as dipole-dipole interactions between individual emitters [13,25-30] or quantum-mechanically by treating the emitters as an interacting spin ensemble coupled to an optical mode [14,31-33]. The latter approach has

\footnotetext{
*hofferberth@sdu.dk

Published by the American Physical Society under the terms of the Creative Commons Attribution 4.0 International license. Further distribution of this work must maintain attribution to the author(s) and the published article's title, journal citation, and DOI.
}

been used to study the propagation of quantized light in onedimensional waveguides, while the semiclassical approach enables investigation of large, weakly driven ensembles in two or three dimensions. In the single-excitation sector and as long as saturation of the medium can be neglected, the two approaches lead to equivalent results $[34,35]$. There has recently been strong interest in structured emitter arrays for tailoring optical properties with unprecedented control [27,28,36,37], for example exploiting subradiance to enhance photon storage fidelities [38,39]. Striking experimental demonstrations of this concept are realizations of highly reflective monolayers with ultracold atoms in optical lattices [40] and solid-state systems $[41,42]$.

In this paper, we study collective directed single-photon emission from an ultracold atomic ensemble which, due to the Rydberg blockade effect [43], can only contain a single Rydberg excitation at any given time and is thus fully saturated by the absorption of a single photon [11,44]. In this limit, the blockaded ensemble reduces to an effective two-level superatom with collectively enhanced coupling to the driving field [45] and enhanced spontaneous decay into the forward direction of the driving mode. In contrast to the previous experimental study of the superatom driven by a few-photon probe [12], we now study with very high accuracy the emitted light in the forward direction after the probe pulse. We experimentally observe that the rate of this collective emission depends on the duration and strength of the initial driving pulse, instead of being solely determined by the number of atoms $N$ forming the superatom. We attribute this effect to a coherent phase evolution of the superatom's collective state that arises from a coherent coupling between degenerate super- and subradiant collective states and leads to a redistribution of population between them. This coupling is mediated by coherent photon exchange between individual atoms inside the superatom. To support this interpretation, we compare our data to an effective model that captures both the coherent internal dynamics as well as the overall dephasing 


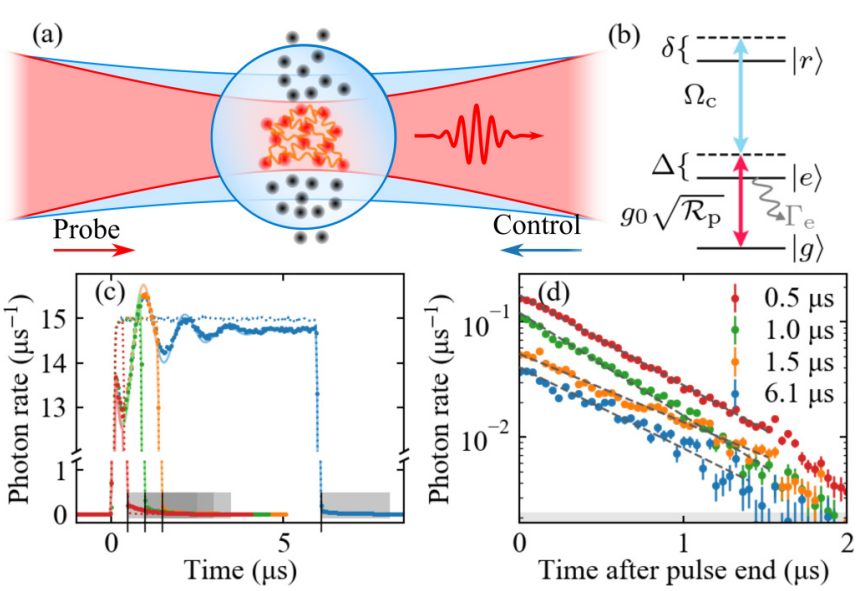

FIG. 1. (a) Sketch of the experimental implementation and (b) single-atom level scheme to create a single Rydberg superatom. (c) Probe pulses of different duration measured with (solid circles) and without atoms (dashed lines). The solid lines show the solution to the master equation for the model system shown in Fig. 3. Shaded areas indicate parts of the pulses included in our analysis. (d) Logarithmic plot of the difference between the signal with and without atoms detected after the driving pulse has ended for different pulse lengths. Gray dashed lines are fits to the data, error-bars are standard error of the mean.

of the collective excitation and find that this simple model captures the observed decay dynamics very well.

\section{EXPERIMENTAL SETUP}

To create a single Rydberg superatom, we optically trap $2 \times 10^{4}{ }^{87} \mathrm{Rb}$ atoms at the foci of counterpropagating probe and control fields [Fig. 1(a) and Appendix A]. The fields, with variable probe photon rate $\mathcal{R}_{\mathrm{p}}$ and control Rabi frequency $\Omega_{\mathrm{c}}=2 \pi \times 13 \mathrm{MHz}$, drive Raman transitions between the ground state $|g\rangle=\left|5 S_{1 / 2}, F=2, m_{F}=2\right\rangle$ and the Rydberg state $|r\rangle=\left|111 S_{1 / 2}, J=1 / 2, m_{J}=1 / 2\right\rangle$ via $|e\rangle=$ $\left|5 P_{3 / 2}, F=3, m_{F}=3\right\rangle$ [Fig. 1(b)]. For large intermediatestate detuning $\Delta,|e\rangle$ can be adiabatically eliminated. Atoms in $|g\rangle$ are thus coupled to $|r\rangle$ with an effective Rabi frequency $\Omega=\sqrt{\mathcal{R}_{\mathrm{p}}} g_{0} \Omega_{\mathrm{c}} /(2 \Delta)$ and Raman decay $\Gamma=\Gamma_{\mathrm{e}}\left(\Omega_{\mathrm{c}} / 2 \Delta\right)^{2}$. Here, $g_{0}$ is the single-photon-single-atom coupling strength and $\Gamma_{\mathrm{e}}=2 \pi \times 6 \mathrm{MHz}$ the natural linewidth of $|e\rangle$. The transmission and re-emission of probe photons in the forward direction is detected behind a single-mode fiber using singlephoton counting modules.

Rydberg blockade limits the ensemble to a single excitation [Fig. 1(a)] and gives rise to its superatom nature with a collective ground state $|G\rangle=\left|g_{1}, \ldots, g_{N}\right\rangle$ and a bright excited state $|W\rangle=\frac{1}{\sqrt{N}} \sum_{j=1}^{N}\left|g_{1}, \ldots, r_{j}, \ldots, g_{N}\right\rangle$, where $N \approx$ 5000 atoms overlap with the probe beam [12]. The coupling between $|G\rangle$ and $|W\rangle$ is collectively enhanced with Rabi frequency $\Omega_{\text {col }}=2 \sqrt{\kappa \mathcal{R}_{\mathrm{p}}}$ and the resulting single-photon coupling $\sqrt{\kappa}=\sqrt{N} g_{0} \Omega_{\mathrm{c}} /(4 \Delta)$ allows us to drive Rabi oscillations with few-photon probe pulses. The single-photon absorption and re-emission into the forward direction can be directly observed in the transmission of the Tukey-shaped probe pulses shown in Fig. 1(c), where $\mathcal{R}_{\mathrm{p}}=15.0 \mu \mathrm{s}^{-1}$ and $\Delta=2 \pi \times 100 \mathrm{MHz}$.
Below, we investigate the collective decay dynamics after extinction of the probe pulse. Towards this end, we vary the pulse duration and record forward-emitted light after extinguishing the probe on a timescale shorter than the enhanced spontaneous decay rate $\kappa$ of the collective state, while the control field remains on. We analyze the photon statistics in the forward direction after the driving pulse stops and obtain for the background-corrected second-order correlation function $g^{(2)}(\tau=0)<0.1$, consistent with single-photon emission. The single-excitation nature of the superatom is further confirmed by the ion-counting statistics obtained after field-ionizing the ensemble at the end of the probe pulse [44]. Besides collective emission, the superatom is also subject to nonradiative dephasing $\gamma_{D}$ of the collectively enhanced state which results in nonretrievable storage of a single photon in the ensemble [44].

Figure 1(d) shows the probe light detected after extinction of the probe field for the four different pulse lengths shown in Fig. 1(c) (following subtraction of residual background light). As expected, we observe an exponential decay, with the initial amplitude determined by the bright-state population. Additionally, though we find that the rate of photon emission changes depending on the probe pulse duration and thus the internal state of the ensemble after the driving pulse. If the superatom's internal collective dynamics were fully incoherent, the decay rate would be constant and given by the sum of single-photon coupling $\kappa$ and the rates for excited-state population loss through Raman decay $\Gamma$ and dephasing $\gamma_{D}$.

\section{SUPERATOM DECAY DYNAMICS}

To systematically study the nonconstant decay rate of the superatom, we repeat the above-described experiment with varying probe pulse length for a range of different parameter sets, varying the single-photon coupling strength $\kappa$ and the probe photon rate $\mathcal{R}_{\mathrm{p}}$ during the pulse (Fig. 2). To quantify the photon decay rate, we assume initially that the decay is exponential and fit the recorded photon rates with $I_{0} e^{-\gamma t}$ to extract $I_{0}$ (here a flux in photons $/ \mu$ s into the probe mode rather than intensity) and the decay rate of forward emission $\gamma$. Because of the single-photon nature of the collective emission, we have to repeat the experiment for each parameter set sufficiently often to obtain mean photon traces as shown in Fig. 1(d). The fits include data points up to $1.5 \mu$ s after the driving pulse ended or up to a threshold where the uncertainty becomes comparable to the absolute value, as discussed in Appendix A (this is shown as shaded regions in Fig. 1).

First, we show in Fig. 2 the obtained amplitudes and rates as function of pulse length for three different values of the intermediate-state detuning $\Delta=2 \pi \times 100 \mathrm{MHz}$ [Figs. 2(a) and 2(e)], $125 \mathrm{MHz}$ [Figs. 2(b) and 2(f)], and $150 \mathrm{MHz}$ [Figs. 2(c) and 2(g)] and a fixed probe photon rate during the pulse of $\mathcal{R}_{\mathrm{p}}=15.0 \mu \mathrm{s}^{-1}$. By changing $\Delta$, we vary both the coupling strength $\kappa$ between $|G\rangle$ and $|W\rangle$, as well as the Raman decay rate $\Gamma$. The initial amplitudes in Figs. 2(e)-2(g) reflect the collective Rabi oscillation of the superatom during the probe pulse. Specifically, the oscillation becomes slower for increasing $\Delta$ since $\Omega \propto 1 / \Delta$. The initial amplitude of the forward emission also reduces over time due to spontaneous Raman decay and dephasing of the collective state. More 

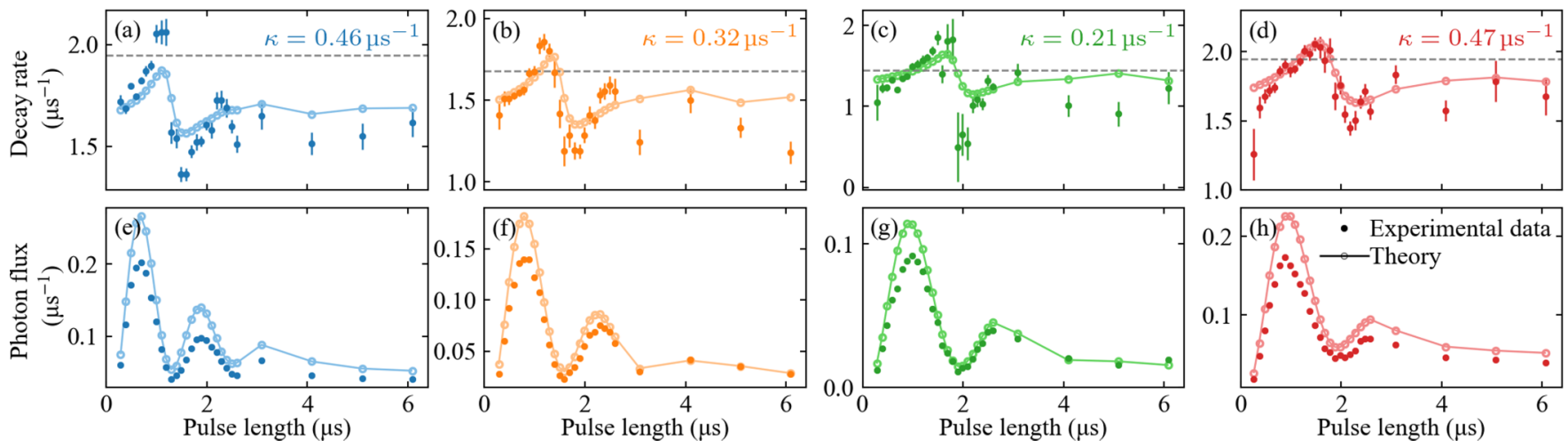

FIG. 2. (a)-(d) Observed decay rates and (e)-(h) initial amplitudes as a function of pulse length extracted from exponential fits to data, as shown in Figs. 1(c) and 1(d) for different values of the single-photon coupling strength $\kappa$ and photon rate $\mathcal{R}_{\mathrm{p}}$. For panels (a)-(c) and (e)-(g) $\mathcal{R}_{\mathrm{p}}=15.0 \mu \mathrm{s}^{-1}$, and for $(\mathrm{d}, \mathrm{h}) \mathcal{R}_{\mathrm{p}}=6.7 \mu \mathrm{s}^{-1}$. In addition to experimental data (dark circles), we show the theoretical results of the extended superatom model discussed in the text and shown in Fig. 3 (empty circles), as well as the constant decay rate of the simple superatom model without internal coherent dynamics (dashed lines). The error bars shown on the rates are one standard deviation confidence intervals of the fits.

surprisingly, we find that, for all data sets the decay rates in Figs. 2(a)-2(c) not only depend on the probe pulse lengths but oscillate out of phase with the amplitude oscillations. The overall magnitude of the rates also reduces with higher $\Delta$ as expected due to the accompanying reduction in $\kappa$ and $\Gamma$. For longer pulse lengths, the decay rate approaches a constant value as the superatom reaches an equilibrium steady state because of dephasing.

We also investigate the effect of changing $\Omega$ by reducing the input probe photon rate $\mathcal{R}_{\mathrm{p}}$ with $\Delta=2 \pi \times 100 \mathrm{MHz}$ fixed. As $\mathcal{R}_{\mathrm{p}}$ is reduced from $15.0 \mu \mathrm{s}^{-1}$, Figs. 2(a) and 2(e), to $6.7 \mu \mathrm{s}^{-1}$, Figs. 2(d) and 2(h), the oscillations in decay rate and initial amplitude become correspondingly slower, but the range of decay rates remains the same. For $\mathcal{R}_{\mathrm{p}}=6.7 \mu \mathrm{s}^{-1}$, $\Omega$ is comparable to the case $\Delta=2 \pi \times 125 \mathrm{MHz}$ and $\mathcal{R}_{\mathrm{p}}=$ $15.0 \mu \mathrm{s}^{-1}$ such that the oscillation periods for both values match, while the decay rates increase, as expected for stronger coupling.

\section{EFFECTIVE FOUR-LEVEL MODEL}

The core conclusion from our observations is that the collective forward emission of the superatom depends on its internal dynamics and the single-excitation state of the ensemble at the end of the driving pulse, which in general is expected to be a superposition of the bright state $|W\rangle$ and subradiant states. In particular, we find a remarkable correlation between the decay rate and the initial intensity, which translates to the probability of being in the bright state. The oscillatory behavior of the observed decay rates indicates that these internal dynamics are based on a coherent process. On a theoretical level, such a coherent term is well understood from a microscopic analysis [19] as the interaction between light and matter not only gives rise to the collective and directed emission, but also to a coherent interaction between the emitters. While in many situations this term can be neglected, it has recently attracted an increased interest [31-33]. In particular, it was demonstrated within a semiclassical approach that this coherent term leads to a nonexponential behavior of the emitted photons [13], as well as a remarkable universal dynamics for a one-dimensional waveguide [14] using the full quantum dynamics.

Next, we demonstrate that an effective four-level model, shown in Fig. 3(a), which incorporates the dynamics of the superatom during the driving pulse, the nontrivial collective decay, as well as the intrinsic coherent coupling between the emitters and the dephasing, is capable to capture the experimentally observed phenomena. The core of the model is a coupling between the collective ground state $|G\rangle$ and the singly excited collective bright state $|W\rangle$ with $\Omega_{\text {col }}$. The coherent coupling of the bright state to $N-1$ other singly excited
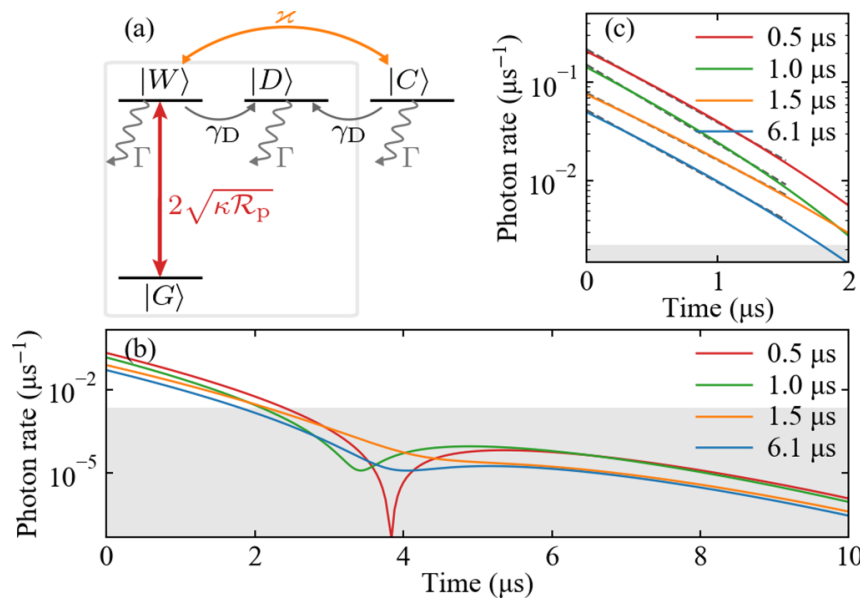

FIG. 3. (a) Level scheme for our model. The probe field couples the collective ground and bright states, $|G\rangle$ and $|W\rangle$, with an effective coupling strength $2 \sqrt{\kappa \mathcal{R}_{\mathrm{p}}}$. The bright state $|W\rangle$ irreversibly decays with rate $\gamma_{\mathrm{D}}$ into a dark state $|D\rangle$, which does not couple to the light. In addition, $|W\rangle$ coherently couples to a single subradiant state $|C\rangle$. This state also decays to $|D\rangle$. All excited states decay via Raman decay $\Gamma$ to $|G\rangle$. (b) Collective emission into the forward direction after the driving pulse has ended as predicted by this model for different pulse lengths calculated for the same parameters as found for the data shown in Figs. 1(c) and 1(d). The shaded area shows the cutoff region of Fig. 1(d). (c) Zoom of panel (b) to the experimentally accessible region shown in Fig. 1(d). The dashed lines show exponential fits. 
subradiant eigenstates due to photon exchange as well as the dephasing of all excited states due to external decoherence are simplified in our model by introducing only two additional states. We model the exchange interactions between all excited states by including a coherent coupling with strength $\varkappa$ between $|W\rangle$ and an additional state denoted as $|C\rangle$. In Appendix B, we demonstrate that, for a chiral waveguide, this approximation is capable to capture with high precision the full dynamics of $N$ atoms. Furthermore, we describe the dephasing of both the bright state $|W\rangle$ and the state $|C\rangle$ by a nonradiative decay into a dark state $|D\rangle$ with rate $\gamma_{D}$. This treatment of the dephasing of the collective excited states is justified for the large number of emitters $N$ in our superatom [46]. Finally, we include the Raman decay of all excited states to the ground state with rate $\Gamma$. Then, the effective model is described by a Lindblad master equation for the superatom density matrix $\rho$ is given by

$$
\begin{aligned}
\partial_{t} \rho(t)= & -\frac{i}{\hbar}\left[H_{0}(t), \rho(t)\right]+(\kappa+\Gamma) \mathcal{D}\left[\sigma_{\mathrm{GW}}\right] \rho(t) \\
& +\gamma_{\mathrm{D}} \mathcal{D}\left[\sigma_{\mathrm{DW}}\right] \rho(t)+\Gamma \mathcal{D}\left[\sigma_{\mathrm{GD}}\right] \rho(t) \\
& +\gamma_{\mathrm{D}} \mathcal{D}\left[\sigma_{\mathrm{DC}}\right] \rho(t)+\Gamma \mathcal{D}\left[\sigma_{\mathrm{GC}}\right] \rho(t),
\end{aligned}
$$

where $\mathcal{D}[\sigma]=\sigma \rho \sigma^{\dagger}-\left(\sigma^{\dagger} \sigma \rho+\rho \sigma^{\dagger} \sigma\right) / 2$ is the Lindblad dissipator. The coherent coupling is described by the Hamiltonian

$$
H_{0}(t)=2 \hbar \sqrt{\kappa \mathcal{R}_{\mathrm{p}}} \sigma_{\mathrm{GW}}^{\dagger}+\hbar \varkappa \sigma_{\mathrm{CW}}^{\dagger}+\text { H.c. }
$$

where $\sigma_{\mu \nu}=|\mu\rangle\langle\nu|$. Note that this four-level model is a natural extension of the previous theoretical study [12], and becomes necessary as we study the emitted light with much higher accuracy.

Equation (1) can be solved numerically and directly compared with the experimental data. Importantly, this simple model describes the superatom Rabi oscillations while the system is driven, while also predicting the time varying collective decay after the driving pulse. Specifically, the predicted decay rate after the probe pulse for the pulse lengths and parameters for the data in Fig. 1 are shown in Fig. 3(b). We find that over the full decay time, the forward emission becomes clearly nonexponential and instead features a drop and revival, which is strongest for the shorter pulses; in agreement with recent predictions in Ref. [13]. This results from coherent excitation shelving into $|C\rangle$ where the excitation is protected from forward emission until it is transferred back into $|W\rangle$. For longer pulses, the feature becomes less prominent as the dynamics are dominated by dephasing, which also prevents the occurrence of a second emission drop. As indicated by the gray-shaded area, the predicted dip in emission occurs at photon rates two orders of magnitude below the experimental noise level. Thus, we focus in Fig. 3(c) on the predicted emission in the experimentally observable early part of the decay. Here, the model already predicts a deviation from purely exponential decay, but comparing with the data [Fig. 1(d)], this tendency in the slope of the emission is not visible at our current experimental resolution.

To compare model and experiment, we extract a decay rate and initial intensity from the numerical solutions as for the experimental data [dashed lines in Fig. 3(c)]. While the nonexponential behavior makes exponential fitting impossible for long times after the pulse has ended, the decay is approximately exponential for the first $1.5 \mu \mathrm{s}$. This way, we obtain the model predictions for the initial slope and intensity of the decay shown in Fig. 2. The qualitative features of the model predictions are robust to small changes of the included time.

In our optimization procedure, we assume that $\gamma_{\mathrm{D}}$ is the same in all datasets and that $\kappa$ scales with the single photon detuning as $\kappa \propto 1 / \Delta^{2}$. The Raman decay rate $\Gamma$ is determined from experimental parameters. The obtained fitting parameters for $\kappa, \varkappa$, and $\gamma_{\mathrm{D}}$ are given in Appendix C. We find that our simple model captures the oscillatory behavior of the decay rate as a function of pulse length quite well for all datasets. Furthermore, the values found for the coherent coupling $\varkappa$ are of the same order as the collective coupling $\kappa$, as expected from the comparison of our model to microscopic simulations of an idealized one-dimensional system, as discussed in Appendix B.

We also show the constant decay rates predicted by the model if we disable the coherent coupling by forcing $\varkappa=0$ (dashed lines in Fig. 2), to show that the coherent coupling is the crucial factor to explain the experimental observations. In previous work, we successfully described single-photon absorption $[44,46]$ and photon correlations mediated by the superatom $[12,47]$ without the coherent coupling. These observations are still captured by the four-level model presented here. However, the influence of the coherent population shelving only becomes visible in the precise study of the decay dynamics after the driving pulse.

\section{CONCLUSION AND OUTLOOK}

In summary, we observed nontrivial collective emission dynamics of a Rydberg superatom, which we attribute to coherent exchange interactions between individual atoms. We show that a simple effective model including a single coherently coupled subradiant state reproduces the experimental observations quite well. Our work is complementary to recent investigations of weakly excited ensembles [13,18,24] and structured emitter arrays [40-42], but adds a new component through the saturation of the ensemble by a single photon because of the Rydberg blockade, which imposes further challenges for a full theoretical treatment. While our model is motivated by comparison to simulations of an idealized waveguide system and captures the core aspects of the system, a full understanding of the coherent and incoherent dynamics in a thermal atomic ensemble will ultimately require a full microscopic model. Our observations are of immediate consequence for the study and application of Rydberg superatoms and other collective quantum emitters, for example in cascaded emitter systems in waveguide-like geometries, where the internal dynamics will significantly alter the behavior of the full ensemble [14]. More generally, these dynamics become relevant whenever collective excitations are created or probed on timescales comparable to the coherent photon exchange rates, e.g., in quantum simulation or photon memories. On one hand, it will be relevant to study to what extent such internal interaction dynamics impose a fundamental limit on applications of collective excitations for single-photon sources and quantum gates. At the same time, a better understanding of these dynamics enable precise collective state 


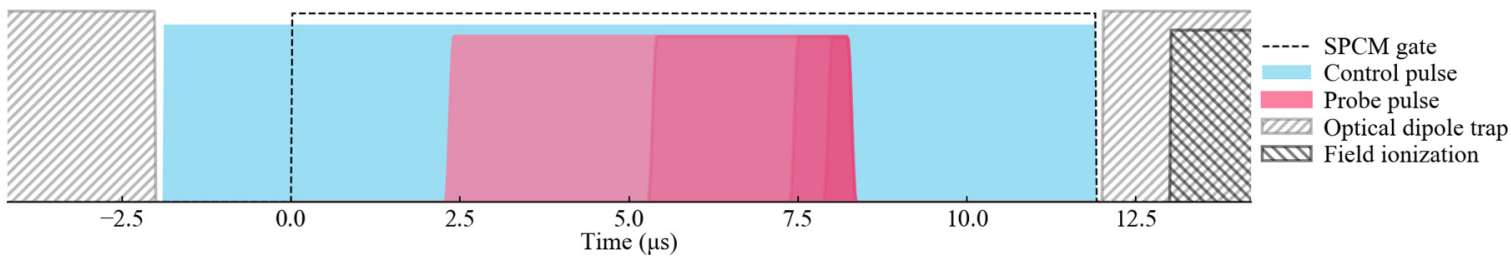

FIG. 4. Sketch of experimental procedure. The crossed optical dipole trap is turned off for $14 \mu \mathrm{s}$. As the dipole trap turns off, the Rydberg control laser is turned on. We employ a $2 \mu \mathrm{s}$ wait time before the gates of the single-photon counter modules are opened to ensure that the control light is fully on. Probing is done during the $12 \mu \mathrm{s}$ where the single-photon counter gates are open. We keep the end-time of the probe pulse fixed and vary the point in time when the probe pulse is turned on. The dipole trap is turned back on as the gates are turned off, and a subsequent field-ionization pulse is applied to remove any remaining Rydberg excitation in the system. Produced ions are detected by a multichannel plate.

engineering, for example to efficiently store photons in subradiant collective states [48].

\section{ACKNOWLEDGMENTS}

We thank Charles Adams, Robert Bettles, Thomas Stolz, Thomas Pohl, and Klaus Mølmer for fruitful discussions. This work is supported by the European Union's Horizon 2020 program under the ERC consolidator grants SIRPOL (Grant No. 681208) and RYD-QNLO (Grant No. 771417), the ErBeStA project (Grant No. 800942), Grant Agreement No. 845218 (Marie Skłodowska-Curie Individual Fellowship to H.B.), and the Deutsche Forschungsgemeinschaft (DFG) under SPP 1929 GiRyd project BU 2247/4-1.

\section{APPENDIX A: EXPERIMENTAL DETAILS}

Below, we give experimental details in addition to the main text, summarizing the ensemble preparation and the experimental sequence that we employ subsequently.

\section{Ensemble preparation}

We start from a cigar-shaped ensemble of ${ }^{87} \mathrm{Rb}$ atoms in a crossed optical dipole (wavelength $1070 \mathrm{~nm}, 1 / e^{2}$ waist $\approx 55 \mu \mathrm{m}$, intersection angle $30^{\circ}$ ) loaded from a magnetooptical trap (MOT). Following a final compression of the MOT, the atoms are evaporatively cooled as we reduce the trap light intensity in two stages. For additional cooling and to reduce atom loss, we employ Raman sideband cooling for $16 \mathrm{~ms}$ during each of the linear evaporation ramps. Longitudinal confinement along the probe axis below the range of Rydberg blockade is provided by a tightly focused optical trap with an elliptical cross section (wavelength $805 \mathrm{~nm}, 1 / e^{2}$ waists $\approx 10 \mu \mathrm{m}$ and $\approx 21 \mu \mathrm{m}$ ) that intersects perpendicularly with the cigar-shaped ensemble as well as the probe and control beams at their focus. In the dimple trap, the ensemble dimensions are $6.5 \mu \mathrm{m}$ along and $21 \mu \mathrm{m} \times 10 \mu \mathrm{m}$ perpendicular to the probe direction $(1 / e$ radii of the Gaussian atomic density distribution) and the atomic temperature is $\approx 10 \mu \mathrm{K}$. The dimple trap intensity is kept constant throughout the evaporation process and the dimple position can be fined tuned with respect to the probe by using an acousto-optical deflector. Before starting experiments, the crossed dipole trap intensity is ramped to zero such that atoms outside the dimple are released and move outside the experimental region before increasing it again to provide confinement in the radial probe direction for the atoms inside the dimple. In combination with the $1 / e^{2}$ waist radius of the probe $(\approx 6.5 \mu \mathrm{m})$, the dimple confinement restricts the excitation volume below the blockade range. The focus of the control beam is larger $(\approx 14 \mu \mathrm{m})$ to limit variation of $\Omega_{c}$ across the excitation volume.

\section{Superatom excitation and probe photon detection}

Following preparation of the atomic ensembles in the dimple, we employ the experimental sequence shown in Fig. 4. The crossed dipole trap is turned off every $100 \mu$ s for $14 \mu \mathrm{s}$. The dimple potential remains present throughout the experiment to restrict motion along the axial probe direction. We account for the resulting AC-Stark shift by adjusting the probe frequency accordingly. The shift also suppresses Rydberg excitation of atoms outside the dimple following release from the crossed-dipole trap.

The control field is turned on as the dipole trap is turned off, and after a $2 \mu s$ wait time the gates of the SPCMs are opened and probing takes place. In the experiments described here, we keep the probe pulse end-time fixed and vary the start time.

Following conclusion of a single experimental shot, an electric-field pulse ionizes any remaining Rydberg atoms to avoid the presence of residual Rydberg excitations during the next iteration of the superatom excitation. The ions are detected on a multichannel plate. Overall, we conduct 1000 individual experimental cycles. Afterward, the atoms are released from the trapping potentials by turning the optical dipole trap and the dimple trap off for $10 \mathrm{~ms}$. The traps are then turned back on and 1000 probing cycles without atoms present are performed to acquire reference pulses of the probe. Subsequently, we prepare a new atomic ensemble by repeating the procedure above.

The light emitted by the superatom is detected by using four single-photon counting modules (SPCMs) in a Hanbury Brown-Twiss configuration located behind a single-mode optical fiber, which is aligned onto the original probe mode and acts as a spatial mode filter. The overall detection efficiency including the SPCM quantum efficiency and all optical loss between the experimental region and the SPCMs is $\approx 35 \%$.

For each of the four datasets presented in the main text, we take data for 26 different pulse lengths. For the dataset with $\Delta=2 \pi \times 100 \mathrm{MHz}, \mathcal{R}_{\mathrm{p}}=15.0$ photons $/ \mu \mathrm{s}$, we take $1111 \times 10^{3}$ measurements for each pulse length. For $\Delta=2 \pi \times 125 \mathrm{MHz}, \mathcal{R}_{\mathrm{p}}=15.0$ photons $/ \mu \mathrm{s}$, we take $621 \times$ 
$10^{3}$ measurements, and for $\Delta=2 \pi \times 150 \mathrm{MHz}, \mathcal{R}_{\mathrm{p}}=$ 15.0 photons $/ \mu$ s we take $467 \times 10^{3}$ measurements. For $\Delta=$ $2 \pi \times 100 \mathrm{MHz}, \mathcal{R}_{\mathrm{p}}=6.7$ photons $/ \mu \mathrm{s}$, we take $377 \times 10^{3}$ measurements.

In the main text, we discuss a threshold on the values included in the fits shown in the main paper in Fig. 1(d). The threshold is the point where the statistical uncertainty on the data points become similar to their values. The threshold is in this case defined as the value where less than 50 counts have been detected in one time-bin of $20 \mathrm{~ns}$ in all the measurements.

\section{APPENDIX B: SUPERATOM MODEL WITH INTERNAL COHERENT DYNAMICS}

In the main text we introduce an effective model with four effective states, see Fig. 3, which attempts to capture both coherent and incoherent internal dynamics of the superatom in the simplest possible way. The main feature of this model is a coherent coupling between a collectively excited bright state and a single collectively excited subradiant state to represent the coherent dynamics inside the superatom, while a second effectively dark state represents weakly coupled collective states into which the system can dephase. We show in the following how this effective model is motivated by the microscopic dynamics of a system of $N$ emitters coupled to a single-mode light field.

Generally, only one excited atomic state $|\mathrm{W}\rangle$ is coupled to the specific mode of the light field and emits collectively back into the same mode. However, $|\mathrm{W}\rangle$ couples coherently to $N-$ 1 subradiant states $\left|C_{i}\right\rangle$ via exchange of virtual photons inside the excited ensemble. The strength of this coherent coupling is different between the different eigenmodes of the system and depends on the individual atom positions. To study the effect of the coherent exchange, we consider a system of $N$ quantum emitters coupled to a one-dimensional chiral waveguide. The chiral one-dimensional waveguide has many similarities to the Rydberg superatom [14] and can be solved analytically in the case of stationary emitters.

In the one-dimensional chiral waveguide, the atoms are coherently coupled to each other by the one-dimensional dipole-dipole interaction

$$
H_{\mathrm{exc}}=\frac{i \hbar \kappa}{2 N} \sum_{l, j} \operatorname{sgn}\left(k_{0}\left(x_{l}-x_{j}\right)\right) \sigma_{l, j},
$$

which describes the exchange of a virtual photon from atom $j$ to atom $l$, mediated by the operator $\sigma_{l, j}$. As stated above, the bright state $|\mathrm{W}\rangle=\sum|j\rangle / \sqrt{N}$, where $|j\rangle$ indicates that the $j$ th atom is in the Rydberg state, is distinct from all other excited states, in that it is the only state coupled to the light field. Therefore, it is practical to reexpress the exchange Hamiltonian in a basis containing the bright state and orthogonal subradiant states. After diagonalization of the subradiant state subspace, the exchange Hamiltonian in this basis becomes

$$
H_{\mathrm{exc}}=\hbar \sum_{j=1}^{N-1}\left(\kappa_{j} \sigma_{\mathrm{W}, \mathrm{C}_{j}}+\text { H.c. }\right)+\hbar \sum_{j=1}^{N-1} \epsilon_{j} \sigma_{\mathrm{C}_{j}, \mathrm{G}} \sigma_{\mathrm{G}, \mathrm{C}_{j}} \text {, }
$$

where $\kappa_{j}=\kappa[i+\cot (\pi j / N)] / 2 N$ and $\epsilon_{j}=-\kappa \cot (\pi j / N) /$ $(2 N)$ are coupling strengths between atomic states. $\left|\mathrm{C}_{j}\right\rangle$ is one of the $N-1$ subradiant states. In this basis we directly see that there are subradiant states with weak coupling to the bright state of order $O(1 / N)$. Another contribution to the dynamics is the driving of the atomic system with incident light with amplitude $\alpha(t)$, which couples the ground state to the bright state,

$$
H_{\text {drive }}=\hbar \sqrt{\kappa} \alpha(t)\left(\sigma_{\mathrm{W}, \mathrm{G}}+\sigma_{\mathrm{G}, \mathrm{W}}\right) .
$$

Here, $\kappa$ is the bright state enhanced coupling to the driving light field. The excited system will eventually decay. However, only the bright state will decay back into the waveguide. Coming from the full microscopic theory, this decay rate is given by $\kappa$. Altogether, the dynamics of the chiral waveguide model are described by the master equation

$$
\partial_{t} \rho=-\frac{i}{\hbar}\left[H_{\mathrm{exc}}+H_{\text {drive }}, \rho\right]+\kappa \mathcal{D}_{\sigma_{\mathrm{G}, \mathrm{W}}}[\rho] .
$$

We can further account for spontaneous decay of single atoms in other directions than the waveguide with a constant rate $\Gamma$. In the bright state-subradiant state basis this adds a decay term $\Gamma \mathcal{D}_{\sigma_{\mathrm{G}, \mathrm{C}_{\mathrm{i}} / \mathrm{W}}}[\rho]$ for each subradiant state and the bright state.
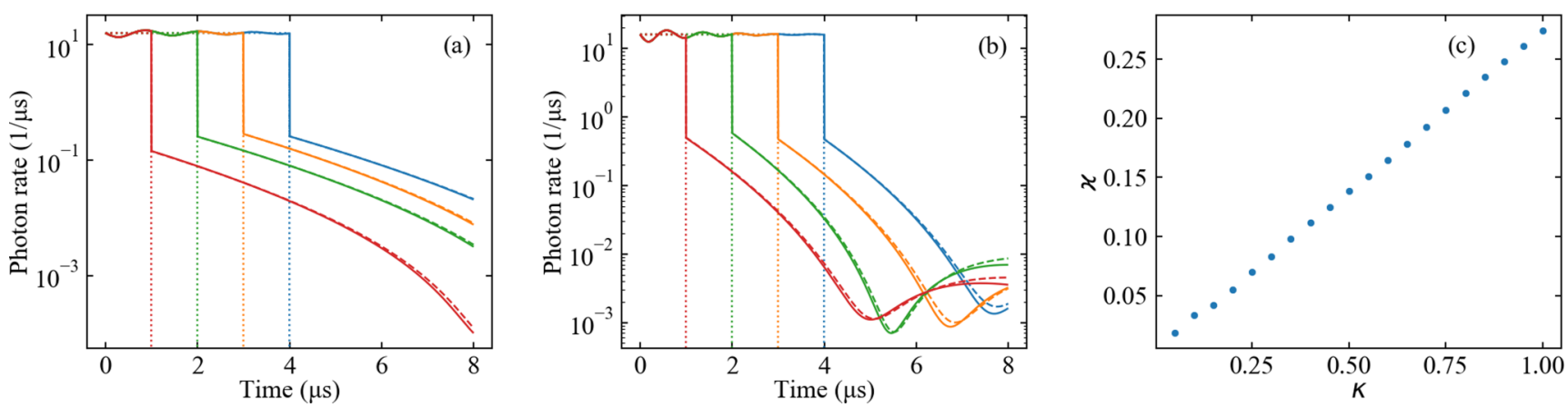

FIG. 5. (a), (b) Emission of light from $N=1000$ atoms coupled to a one-dimensional waveguide for four pulse lengths and with (a) $\kappa=$ $0.45 \mu \mathrm{s}^{-1}$ and (b) $\kappa=1.0 \mu \mathrm{s}^{-1}$. Solid lines are results from the waveguide, dashed lines are fits with the four-level model to the waveguide results, and dotted lines show where the driving field ends. $\Gamma=0.1 \mu \mathrm{s}^{-1}$. Comparing with experimental data, the value of $\kappa$ in panel (a) is the same as we find in the experiment, whereas panel (b) shows a higher $\kappa$ but $\varkappa$ similar to what is found in experiment. (c) By fitting the four-level model to the chiral waveguide data for different values of $\kappa$ it is possible to extract a scaling of $\varkappa$ with $\kappa$. 
Solving the master equation of the chiral waveguide model (B4), we can calculate the light emission from $N=1000$ emitters for varying pulse lengths and for different $\kappa$, with examples shown by the solid lines in Figs. 5(a) and 5(b). We find that these calculations indeed show varying forward-directed decay of the ensemble, as observed in the experiment.

Next, we can test how well our simplified effective model with only a single coherently coupled state reproduces these results. Towards this end, we fit the numerical calculations with our effective model in the same way as we fit the experimental data, with $\Gamma$ fixed to the calculated Raman lifetime to obtain the model parameters $\kappa$ and $\varkappa$. The best fits of the effective model are shown as dashed lines in Figs. 5(a) and 5(b). We find that reducing the $N-1$ collective subradiant states to a single coherently coupled level works extremely well for the chiral waveguide case. We can understand this from the above observation that only a few subradiant states have sufficiently strong coupling to the bright state to contribute significantly to the coherent part of the dynamics on the timescale of the collective decay. Moreover, from analyzing simulations with different collective coupling strength to the waveguide, we find a linear scaling between $\kappa$ and $\varkappa$ for the best fits of our model, as shown in Fig. 5(c).

The considered system of stationary emitters in the chiral one-dimensional waveguide theory contains no sources of dephasing or decoherence except the single-atom spontaneous decay. In the experiment, multiple sources contribute to finite decoherence on the relevant timescale. First, we assume that thermal motion plays a crucial role. For our ensemble temperature $T=10 \pm 1 \mu \mathrm{K}$, we estimate that the atoms have a most likely velocity of $v \approx 0.035 \lambda / \mu \mathrm{s}$, where $\lambda=2 \pi\left(\frac{1}{k_{\mathrm{p}}}-\frac{1}{k_{\mathrm{c}}}\right)$ is the spin wavelength imprinted by the light into the ensemble. $k_{\mathrm{p}}$ and $k_{\mathrm{c}}$ are the wave vectors of the probe and the control fields, respectively. The sign difference arises because the two fields are counterpropagating. This means that, during one measurement, the atoms move about half of a spin-wavelength. Adding thermal motion to our onedimensional model in the form of Boltzmann distributed velocity for each atom does not alter the dynamics significantly. This is specific for the chiral waveguide, since here the time-dependent phase each atom picks up because of its velocity can be transformed into a detuning, effectively doubling the Doppler shift. This is not possible in a nonchiral or three-dimensional system. With this in mind, thermal motion may be added by an effective dephasing model, mixing the excited states with each other. Other sources of dephasing in the experiment can originate from finite laser linewidth or fluctuating electric fields in the experiment chamber.
TABLE I. Parameters sets for theory data.

\begin{tabular}{lcccccc}
\hline \hline $\begin{array}{l}\mathcal{R} \\
\left(\mu \mathrm{s}^{-1}\right)\end{array}$ & $\begin{array}{c}\Delta / 2 \pi \\
\left(\mu \mathrm{s}^{-1}\right)\end{array}$ & $\begin{array}{c}\kappa \\
\left(\mu \mathrm{s}^{-1}\right)\end{array}$ & $\begin{array}{c}\Gamma \\
\left(\mu \mathrm{s}^{-1}\right)\end{array}$ & $\begin{array}{c}\gamma_{\mathrm{D}} \\
\left(\mu \mathrm{s}^{-1}\right)\end{array}$ & $\begin{array}{c}\varkappa \\
\left(\mu \mathrm{s}^{-1}\right)\end{array}$ & $\begin{array}{c}\text { In } \\
\text { figures }\end{array}$ \\
\hline 15.0 & 100 & 0.46 & 0.15 & 0.85 & 0.31 & $1,2,3$ \\
15.0 & 125 & 0.32 & 0.10 & 0.85 & 0.32 & 2 \\
15.0 & 150 & 0.21 & 0.064 & 0.85 & 0.31 & 2 \\
6.7 & 100 & 0.47 & 0.15 & 0.85 & 0.34 & 3 \\
\hline \hline
\end{tabular}

We thus need to include additional dephasing in our effective model and the chiral waveguide model to reproduce the experimental results. Dephasing effectively shifts population from the bright state and the few strongly coupled subradiant states into the many weakly coupled subradiant states. For large atom number $N$, this can be treated as an effective decay into the uncoupled states [46] because no form of revival will happen on the experiment timescale [44]. We implement this in our effective model by adding an additional dark state that acts as a reservoir into which both bright and subradiant state can decay with rate $\gamma_{D}$.

\section{APPENDIX C: EXPERIMENT PARAMETER ESTIMATION}

Here, we outline the procedure used to obtain the parameters used for the results of the effective four-level model presented in Fig. 2. First, we fix $\Gamma=\Gamma_{\mathrm{e}}\left(\Omega_{\mathrm{c}} / 2 \Delta\right)^{2}$ based on the known values for $\Delta$ and $\Omega_{\mathrm{c}}=2 \pi \times 13 \mathrm{MHz}$. We then fit the four-level model with $\varkappa=0$ to the full probe pulse as in previous works [12] to extract $\kappa$ and verify that $\kappa \sim 1 / \Delta^{2}$ for the different datasets. To determine $\gamma_{D}$ and $\varkappa$, the full four-level model shown in Fig. 3(a) is fit to the data. Since the fits put more weight on the points within the pulses, the values found for $\gamma_{D}$ tend to be overestimated. We attribute this overestimation to additional decoherence channels which the model does not account for, such as laser linewidth. Therefore, $\gamma_{\mathrm{D}}$ is adjusted separately to reproduce the observed emission rate after the probe pulse is over. We find a common value for all datasets since dephasing is dominated by atomic motion, which is independent of $\mathcal{R}_{\mathrm{p}}$ and $\Delta$. Table I lists the full parameter sets for the theory data in Fig. 2.

Interestingly, we find approximately the same value of $\varkappa$ for the three different values of $\kappa$ discussed in this work. In contrast to the previously discussed one-dimensional chiral waveguide model we do not find a scaling of $\varkappa$ with $\kappa$ in the experiment.
[1] R. H. Dicke, Coherence in spontaneous radiation processes, Phys. Rev. 93, 99 (1954)

[2] M. Gross and S. Haroche, Superradiance: An essay on the theory of collective spontaneous emission, Phys. Rep. 93, 301 (1982).

[3] R. G. DeVoe and R. G. Brewer, Observation of Superradiant and Subradiant Spontaneous Emission of Two Trapped Ions, Phys. Rev. Lett. 76, 2049 (1996).
[4] C. Hettich, C. Schmitt, J. Zitzmann, S. Kühn, I. Gerhardt, and V. Sandoghdar, Nanometer resolution and coherent optical dipole coupling of two individual molecules, Science 298, 385 (2002).

[5] A. F. van Loo, A. Fedorov, K. Lalumière, B. C. Sanders, A. Blais, and A. Wallraff, Photon-mediated interactions between distant artificial atoms, Science 342, 1494 (2013). 
[6] M. Scheibner, T. Schmidt, L. Worschech, A. Forchel, G. Bacher, T. Passow, and D. Hommel, Superradiance of quantum dots, Nat. Phys. 3, 106 (2007).

[7] P. Tighineanu, R. S. Daveau, T. B. Lehmann, H. E. Beere, D. A. Ritchie, P. Lodahl, and S. Stobbe, Single-Photon Superradiance from a Quantum Dot, Phys. Rev. Lett. 116, 163604 (2016).

[8] R. Röhlsberger, K. Schlage, B. Sahoo, S. Couet, and R. Rüffer, Collective Lamb shift in single-photon superradiance, Science 328, 1248 (2010).

[9] W. Guerin, M. O. Araújo, and R. Kaiser, Subradiance in a Large Cloud of Cold Atoms, Phys. Rev. Lett. 116, 083601 (2016).

[10] S. D. Jenkins, J. Ruostekoski, N. Papasimakis, S. Savo, and N. I. Zheludev, Many-Body Subradiant Excitations in Metamaterial Arrays: Experiment and Theory, Phys. Rev. Lett. 119, 053901 (2017).

[11] Y. O. Dudin and A. Kuzmich, Strongly interacting Rydberg excitations of a cold atomic gas, Science 336, 887 (2012).

[12] A. Paris-Mandoki, C. Braun, J. Kumlin, C. Tresp, I. Mirgorodskiy, F. Christaller, H. P. Büchler, and S. Hofferberth, Free-Space Quantum Electrodynamics with a Single Rydberg Superatom, Phys. Rev. X 7, 041010 (2017).

[13] R. J. Bettles, T. Ilieva, H. Busche, P. Huillery, S. W. Ball, N. L. R. Spong, and C. S. Adams, Collective mode interferences in light-matter interactions, arXiv:1808.08415.

[14] J. Kumlin, S. Hofferberth, and H. P. Büchler, Emergent Universal Dynamics for an Atomic Cloud Coupled to an Optical Waveguide, Phys. Rev. Lett. 121, 013601 (2018).

[15] M. Chalony, R. Pierrat, D. Delande, and D. Wilkowski, Coherent flash of light emitted by a cold atomic cloud, Phys. Rev. A 84, 011401(R) (2011).

[16] C. C. Kwong, T. Yang, M. S. Pramod, K. Pandey, D. Delande, R. Pierrat, and D. Wilkowski, Cooperative Emission of a Coherent Superflash of Light, Phys. Rev. Lett. 113, 223601 (2014).

[17] S. L. Bromley, B. Zhu, M. Bishof, X. Zhang, T. Bothwell, J. Schachenmayer, T. L. Nicholson R. Kaiser, S. F. Yelin, M. D. Lukin, A. M. Rey, and J. Ye, Collective atomic scattering and motional effects in a dense coherent medium, Nat. Commun. 7, 11039 (2016).

[18] S. Jennewein, L. Brossard, Y. R. P. Sortais, A. Browaeys, P. Cheinet, J. Robert, and P. Pillet, Coherent scattering of nearresonant light by a dense, microscopic cloud of cold two-level atoms: Experiment versus theory, Phys. Rev. A 97, 053816 (2018).

[19] R. H. Lehmberg, Radiation from an $N$-atom system. I. General formalism, Phys. Rev. A 2, 883 (1970).

[20] R. Friedberg, S. R. Hartmann, and J. T. Manassah, Frequency shifts in emission and absorption by resonant systems of twolevel atoms, Phys. Rep. 7, 101 (1973).

[21] M. O. Scully, Collective Lamb Shift in Single Photon Dicke Superradiance, Phys. Rev. Lett. 102, 143601 (2009).

[22] Z. Meir, O. Schwartz, E. Shahmoon, D. Oron, and R. Ozeri, Cooperative Lamb Shift in a Mesoscopic Atomic Array, Phys. Rev. Lett. 113, 193002 (2014).

[23] S. J. Roof, K. J. Kemp, M. D. Havey, and I. M. Sokolov, Observation of Single-Photon Superradiance and the Cooperative Lamb Shift in an Extended Sample of Cold Atoms, Phys. Rev. Lett. 117, 073003 (2016).

[24] T. Peyrot, Y. R. P. Sortais, A. Browaeys, A. Sargsyan, D. Sarkisyan, J. Keaveney, I. G. Hughes, and C. S. Adams, Col- lective Lamb Shift of a Nanoscale Atomic Vapor Layer Within a Sapphire Cavity, Phys. Rev. Lett. 120, 243401 (2018).

[25] B. Hopkins, A. N. Poddubny, A. E. Miroshnichenko, and Y. S. Kivshar, Revisiting the physics of Fano resonances for nanoparticle oligomers, Phys. Rev. A 88, 053819 (2013).

[26] R. J. Bettles, S. A. Gardiner, and C. S. Adams, Cooperative ordering in lattices of interacting two-level dipoles, Phys. Rev. A 92, 063822 (2015).

[27] R. J. Bettles, S. A. Gardiner, and C. S. Adams, Enhanced Optical Cross Section via Collective Coupling of Atomic Dipoles in a 2D Array, Phys. Rev. Lett. 116, 103602 (2016).

[28] E. Shahmoon, D. S. Wild, M. D. Lukin, and S. F. Yelin, Cooperative Resonances in Light Scattering from Two-Dimensional Atomic Arrays, Phys. Rev. Lett. 118, 113601 (2017).

[29] A. Asenjo-Garcia, J. D. Hood, D. E. Chang, and H. J. Kimble, Atom-light interactions in quasi-one-dimensional nanostructures: A Green's-function perspective, Phys. Rev. A 95, 033818 (2017).

[30] W. Guerin and R. Kaiser, Population of collective modes in light scattering by many atoms, Phys. Rev. A 95, 053865 (2017).

[31] T. Caneva, M. T. Manzoni, T. Shi, J. S. Douglas, J. I. Cirac, and D. E. Chang, Quantum dynamics of propagating photons with strong interactions: A generalized input-output formalism, New J. Phys. 17, 113001 (2015).

[32] H. Pichler, T. Ramos, A. J. Daley, and P. Zoller, Quantum optics of chiral spin networks, Phys. Rev. A 91, 042116 (2015).

[33] B. Zhu, J. Cooper, J. Ye, and A. M. Rey, Light scattering from dense cold atomic media, Phys. Rev. A 94, 023612 (2016).

[34] A. A. Svidzinsky, J.-T. Chang, and M. O. Scully, Cooperative spontaneous emission of $N$ atoms: Many-body eigenstates, the effect of virtual Lamb shift processes, and analogy with radiation of $N$ classical oscillators, Phys. Rev. A 81, 053821 (2010).

[35] E. Shahmoon and G. Kurizki, Nonlinear theory of laser-induced dipolar interactions in arbitrary geometry, Phys. Rev. A 89, 043419 (2014).

[36] S. D. Jenkins and J. Ruostekoski, Controlled manipulation of light by cooperative response of atoms in an optical lattice, Phys. Rev. A 86, 031602(R) (2012).

[37] R. Bekenstein, I. Pikovski, H. Pichler, E. Shahmoon, S. F. Yelin, and M. D. Lukin, Quantum metasurfaces with atom arrays, Nat. Phys. 16, 676 (2020).

[38] A. Asenjo-Garcia, M. Moreno-Cardoner, A. Albrecht, H. J. Kimble, and D. E. Chang, Exponential Improvement In Photon Storage Fidelities Using Subradiance and "Selective Radiance" in Atomic Arrays, Phys. Rev. X 7, 031024 (2017).

[39] G. Facchinetti, S. D. Jenkins, and J. Ruostekoski, Storing Light with Subradiant Correlations in Arrays of Atoms, Phys. Rev. Lett. 117, 243601 (2016).

[40] J. Rui, D. Wei, A. Rubio-Abadal, S. Hollerith, J. Zeiher, D. M. Stamper-Kurn, C. Gross, and I. Bloch, A subradiant optical mirror formed by a single structured atomic layer, Nature (London) 583, 369 (2020).

[41] P. Back, S. Zeytinoglu, A. Ijaz, M. Kroner, and A. Imamoğlu, Realization of an Electrically Tunable Narrow-Bandwidth Atomically Thin Mirror Using Monolayer $\mathrm{MoSe}_{2}$, Phys. Rev. Lett. 120, 037401 (2018).

[42] G. Scuri, Y. Zhou, A. A. High, D. S. Wild, C. Shu, K. De Greve, L. A. Jauregui, T. Taniguchi, K. Watanabe, P. Kim, M. D. Lukin, and H. Park, Large Excitonic Reflectivity of Monolayer $\mathrm{MoSe}_{2}$ 
Encapsulated in Hexagonal Boron Nitride, Phys. Rev. Lett. 120, 037402 (2018).

[43] M. D. Lukin, M. Fleischhauer, R. Cote, L. M. Duan, D. Jaksch, J. I. Cirac, and P. Zoller, Dipole Blockade and Quantum Information Processing in Mesoscopic Atomic Ensembles, Phys. Rev. Lett. 87, 037901 (2001).

[44] C. Tresp, C. Zimmer, I. Mirgorodskiy, H. Gorniaczyk, A. ParisMandoki, and S. Hofferberth, Single-Photon Absorber Based on Strongly Interacting Rydberg Atoms, Phys. Rev. Lett. 117, 223001 (2016).

[45] Y. O. Dudin, L. Li, F. Bariani, and A. Kuzmich, Observation of coherent many-body Rabi oscillations, Nat. Phys. 8, 790 (2012).
[46] J. Honer, R. Löw, H. Weimer, T. Pfau, and H. P. Büchler, Artificial Atoms Can Do More than Atoms: Deterministic Single Photon Subtraction from Arbitrary Light Fields, Phys. Rev. Lett. 107, 093601 (2011).

[47] N. Stiesdal, J. Kumlin, K. Kleinbeck, P. Lunt, C. Braun, A. Paris-Mandoki, C. Tresp, H. P. Büchler, and S. Hofferberth, Observation of Three-Body Correlations for Photons Coupled to a Rydberg Superatom, Phys. Rev. Lett. 121, 103601 (2018).

[48] M. T. Manzoni, M. Moreno-Cardoner, A. Asenjo-Garcia, J. V. Porto, A. V. Gorshkov, and D. E. Chang, Optimization of photon storage fidelity in ordered atomic arrays, New J. Phys. 20, 083048 (2018). 